\title{
ON TWO CLASSICAL RESULTS OF I. SCHUR
}

\author{
BY MARVIN MARCUS ${ }^{1}$ \\ Communicated by A. S. Householder, May 11, 1964
}

1. Introduction. The results referred to in the title of this announcement are contained in [3]. A detailed statement of them follows.

TheOREM 1. Let $G$ be a subgroup of the symmetric group $S_{n}$ of degree $n$. Let $M$ be a representation of $G$ as unitary linear operators on some unitary space $U, \operatorname{dim} U=m$, and let $H=\left(h_{i j}\right)$ be an $n$-square positive definite hermitian matrix. Then

$$
M_{H}=\sum_{\sigma \in G} M(\sigma) \prod_{i=1}^{n} h_{i \sigma(i)}
$$

is a positive definite hermitian transformation on $U$.

Let $M$ be an arbitrary representation of $G$ as linear operators on some unitary space $U, \operatorname{dim} U=m$, and let $\chi$ be the character of $M$. Define the generalized matrix function $d_{x}(H)$ by

$$
d_{\chi}(H)=\operatorname{tr}\left(M_{H}\right)=\sum_{\sigma \in G} \chi(\sigma) \prod_{i=1}^{n} h_{i \sigma(i)} .
$$

Schur's second result relates $d_{\chi}(H)$ to the determinant of $H$.

THeorem 2. If $H$ is an $n$-square positive semi-definite hermitian matrix then

$$
d_{x}(H) \geqq m \operatorname{det} H .
$$

As Schur points out, the inequality (1.3) contains the Hadamard determinant theorem and the Fischer inequality as special cases. Schur's proofs of Theorems 1 and 2 are lengthy and extraordinarily intricate. The purpose of this announcement is to prove Theorem 3 below. This result contains Theorems 1 and 2 as corollaries and constitutes a substantial generalization of them both. Moreover, the proof outlined here is quite simple and direct. The technique is also currently producing new inequalities for the generalized matrix functions $d_{x}$.

TheOREM 3. Let $G$ be a subgroup of the symmetric group $S_{n}$ of degree $n$. Let $M$ be a representation of $G$ as unitary linear operators on a finite

${ }^{1}$ Supported by NSF Grant GP-1085. Part of this work was completed during the 1964 Gatlinburg Matrix Symposium. 
dimensional unitary space $U, \operatorname{dim} U=m$. For $A$ an arbitrary $n$-square matrix define $M_{A}$ by

$$
M_{A}=\sum_{\sigma \in G} M(\sigma) \prod_{i=1}^{n} a_{i \sigma(i)} .
$$

If $x$ and $y$ are any two vectors in $U$ and $A$ and $B$ are any two $n$-square matrices then

$$
\left|\left(M_{A B} x, y\right)\right|^{2} \leqq\left(M_{A A^{*} x} x\right)\left(M_{B^{*} B} y, y\right) .
$$

To obtain Theorems 1 and 2 from the inequality (1.5) set $B=I_{n}$ and $H=A^{*} A$ where $A$ is a triangular matrix. Then $M_{I_{n}}=I_{m}$ and $M_{A}=\prod_{i=1}^{n} a_{i i} I_{m}=(\operatorname{det} A) I_{m}$. With $x=y$ and $\|x\|=1$ in (1.5) we immediately obtain

(1.6) $\operatorname{det} H=|\operatorname{det} A|^{2}=\left|\left(M_{A} x, x\right)\right|^{2} \leqq\left(M_{A A^{*}} x, x\right)=\left(M_{H} x, x\right)$.

We also compute immediately from the definition that for any $n$ square $X,\left(M_{X}\right)^{*}=M_{X^{*}}$ and hence $M_{H}$ is hermitian when $H$ is. It follows from (1.6) that if $H$ is positive definite then $M_{H}$ is positive definite as well. Moreover, every eigenvalue of $M_{H}$ is bounded below by $\operatorname{det} H$ and thus

$$
d_{x}(H)=\operatorname{tr}\left(M_{H}\right) \geqq m \operatorname{det} H,
$$

the inequality (1.3).

It is worth remarking that the inequality (1.5) can be seen to imply for any $A$ and $B$ that

$$
\left|d_{x}(A B)\right|^{2} \leqq d_{x}\left(A A^{*}\right) d_{x}\left(B^{*} B\right) .
$$

The inequality (1.7) was previously known in case $\chi$ is a character of degree 1 . In fact, this result is the starting point of a sequence of results on the function $d_{x}[1],[2]$.

2. Proof outline. To prove Theorem 3 we need some elementary results about tensor spaces.

Let $V_{1}, \cdots, V_{n}$ be finite dimensional unitary spaces over the complex numbers and let $\otimes_{i=1}^{n} V_{i}$ denote their tensor product. If $x_{i} \in V_{i}$, $i=1, \cdots, n$, then $x_{1} \otimes \cdots \otimes x_{n}=f$ is the tensor in $\otimes_{i=1}^{n} V_{i}$ whose value on any $n$-multilinear functional $\phi$ is $f(\phi)=\phi\left(x_{1}, \cdots, x_{n}\right)$. We won't notationally distinguish the inner products in the various $V_{i}$. An inner product in $\otimes_{i=1}^{n} V_{i}$ is given by

$$
\left(x_{1} \otimes \cdots \otimes x_{n}, y_{1} \otimes \cdots \otimes y_{n}\right)=\prod_{i=1}^{n}\left(x_{i}, y_{i}\right) .
$$


If $A_{i}$ is a linear transformation on $V_{i}, i=1, \cdots, n$, then the tensor product of the $A_{i}$ is a linear transformation on $\otimes_{i=1}^{n} V_{i}$ defined by the formula

$$
A_{1} \otimes \cdots \otimes A_{n} x_{1} \otimes \cdots \otimes x_{n}=A_{1} x_{1} \otimes \cdots \otimes A_{n} x_{n} .
$$

The formulas

$$
\left(A_{1} \otimes \cdots \otimes A_{n}\right)\left(B_{1} \otimes \cdots \otimes B_{n}\right)=A_{1} B_{1} \otimes \cdots \otimes A_{n} B_{n}
$$

and

$$
\left(A_{1} \otimes \cdots \otimes A_{n}\right)^{*}=A_{1}^{*} \otimes \cdots \otimes A_{n}{ }^{*}
$$

are immediate consequences of (2.1) and (2.2). The star on the left side of (2.4) is the conjugate dual with respect to the inner product defined in (2.1) and the star on $A_{i}$ is the conjugate dual with respect to the inner product in $V_{i}$. If $V_{1}=\cdots=V_{n}=V$, and $\sigma \in S_{n}$ then the permutation operators $P(\sigma)$ defined by

$$
P(\sigma) x_{1} \otimes \cdots \otimes x_{n}=x_{\phi(1)} \otimes \cdots \otimes x_{\phi(n)}, \quad \phi=\sigma^{-1},
$$

constitute a representation of $G$ as unitary operators on $\otimes_{i=1}^{n} V$. Set $W=\otimes_{i=1}^{n} V$ and define the linear transformation $T$ on $U \otimes W$ by

$$
T=\sum_{\sigma \in G} M(\sigma) \otimes P(\sigma) .
$$

It is trivial to verify the following statement:

$T$ is hermitian and essentially idempotent. That is,

$$
T^{*}=T, \quad T^{2}=g T
$$

where $g$ is the order of $G$.

Next let $x, y \in U$, and $v, w \in W$. From the Cauchy-Schwarz inequality applied to the inner product in $U \otimes W$ we obtain

$$
|(T x \otimes v, T y \otimes w)|^{2} \leqq(T x \otimes v, T x \otimes v)(T y \otimes w, T y \otimes w)
$$

and from the hermitian idempotent properties of $T$ we obtain

$$
|(T x \otimes v, y \otimes w)|^{2} \leqq(T x \otimes v, x \otimes v)(T y \otimes w, y \otimes w) .
$$

From (2.7) and (2.8) it follows that

$$
\begin{aligned}
& \left|\left(\sum_{\sigma \in G}(P(\sigma) v, w) M(\sigma) x, y\right)\right|^{2} \\
& \quad \leqq\left(\sum_{\sigma \in G}(P(\sigma) v, v) M(\sigma) x, x\right)\left(\sum_{\sigma \in G}(P(\sigma) w, w) M(\sigma) y, y\right) .
\end{aligned}
$$


Set $v=v_{1} \otimes \cdots \otimes v_{n}, w=w_{1} \otimes \cdots \otimes w_{n}$ where $v_{i}, w_{i} \in V, i=1, \cdots, n$, and observe that

$$
(P(\sigma) v, w)=\prod_{i=1}^{n}\left(v_{i}, w_{\sigma(i)}\right)
$$

Thus (2.9) becomes

$$
\begin{aligned}
& \left|\left(\sum_{\sigma \in G} \prod_{i=1}^{n}\left(v_{i}, w_{\sigma(i)}\right) M(\sigma) x, y\right)\right|^{2} \\
& \quad \leqq\left(\sum_{\sigma \in G} \prod_{i=1}^{n}\left(v_{i}, v_{\sigma(i)}\right) M(\sigma) x, x\right)\left(\sum_{\sigma \in G} \prod_{i=1}^{n}\left(w_{1}, w_{\sigma(i)}\right) M(\sigma) y, y\right) .
\end{aligned}
$$

We now specialize $V$ to be the space of $n$-tuples over the complex numbers with the usual inner product. If $A$ and $B$ are any $n$-square matrices let $v_{1}, \cdots, v_{n}$ be the rows of $A$ and $\bar{w}_{1}, \cdots, \bar{w}_{n}$ be the columns of $B$ so that the $(i, j)$ entry of $A B$ is just $\left(v_{i}, w_{j}\right)$. Then (2.10) immediately yields (1.5).

\section{REFERENCES}

1. Marvin Marcus and Morris Newman, Inequalities for the permanent function, Ann. of Math. (2) 75 (1962), 47-62.

2. Marvin Marcus and Henryk Minc, Inequalities for general matrix functions, Bull. Amer. Math. Soc. 70 (1964), 308-313.

3. I. Schur, Über endliche Gruppen und Hermitesche Formen, Math. Z. 1 (1918), 184-207.

University of California, Santa Barbara 